\title{
Dose prediction accuracy of collapsed cone convolution superposition algorithm in a multi-layer inhomogenous phantom
}

\author{
Stephen Oyewale \\ Department of Radiation Oncology, Cancer Centers of Southwest Oklahoma, Lawton, Oklahoma, USA.
}

Received September 01, 2013; Revised September 17, 2013; Accepted September 18, 2013; Published Online September 18, 2013

\section{Original Article}

\begin{abstract}
Purpose: Dose prediction accuracy of dose calculation algorithms is important in external beam radiation therapy. This study investigated the effect of air gaps on depth dose calculations computed by collapsed cone convolution superposition (CCCS) algorithm. Methods: A computed tomography (CT) scan of inhomogenous phantom $\left(30 \times 30 \times 30 \mathrm{~cm}^{3}\right)$ containing rectangular solid-water blocks and two $5 \mathrm{~cm}$ air gaps was used for central axis dose calculations computed by CCCS in Pinnacle treatment planning system. Depth dose measurements were taken using a cylindrical ionization chamber for identical beam parameters and monitor units as in the depth dose computations. The calculated and the measured percent depth dose (PDDs) were then compared. The data presented in this study included $6 \mathrm{MV}$ photon beam and field sizes of $3 \times 3 \mathrm{~cm}^{2}, 5 \times 5 \mathrm{~cm}^{2}, 10 \times 10 \mathrm{~cm}^{2}$, and $15 \times 15 \mathrm{~cm}^{2}$. Results: The results of CCCS were within $\pm 1.4 \%$ in the first water medium. However, upon traversing the first air gap and re-entering the water medium, in comparison to the measurements, the CCCS under-predicted the dose, with difference ranged from $-1.6 \%$ to $-3.3 \%$ for $3 \times 3 \mathrm{~cm}^{2}$, from $-2.4 \%$ to $-4.2 \%$ for $5 \times 5 \mathrm{~cm}^{2}$, from $-2.4 \%$ to $-6.7 \%$ for $10 \times 10 \mathrm{~cm}^{2}$, and from $-1.6 \%$ to $-6.3 \%$ for $15 \times 15 \mathrm{~cm}^{2}$. After the second air gap, the CCCS continued to under-predict the dose, and the difference ranged from $-3.2 \%$ to $-3.9 \%$ for $3 \times 3 \mathrm{~cm}^{2}$, from $-2.4 \%$ to $-5.6 \%$ for $5 \times 5 \mathrm{~cm}^{2}$, from $-2.3 \%$ to $-6.0 \%$ for $10 \times 10 \mathrm{~cm}^{2}$, and from $-1.5 \%$ to $-5.6 \%$ for $15 \times 15 \mathrm{~cm}^{2}$. Conclusion: The CCCS under-predicted the dose in water medium after the photon beam traversed the air gap. Special attention must be given during the patient set-up since large air gap between the patient body and immobilization devices may lead to unacceptable dose prediction errors.
\end{abstract}

Keywords: Collapsed Cone Convolution Superposition; Heterogeneity Correction; PDD; Pinnacle; Dose Calculation

\section{Introduction}

The significant advances in external beam radiation therapy (EBRT) such as beam delivery capabilities have improved the dose conformity and distributions. ${ }^{1}$ The intensity modulation radiation therapy (IMRT) is an example of EBRT that combines several intensity modulated beams leading to the con-

Corresponding author: Stephen Oyewale; Department of Radiation Oncology, Cancer Centers of Southwest Oklahoma, Lawton, Oklahoma, USA. Email: steve.oyewale@gmail.com

Cite this article as:

Oyewale S. Dose prediction accuracy of collapsed cone convolution superposition algorithm in a multi-layer inhomogenous phantom. Int J Cancer Ther Oncol 2013; 1(1):01016. DOI: $10.14319 /$ ijcto.0101.6

A part of this research was presented at $52^{\text {nd }}$ annual meeting of American Association of Physicists in Medicine (AAPM), which was held from August 4-8, 2013 in Indianapolis, Indiana, USA. struction of conformal dose distributions. ${ }^{2,3}$ Most recently, a novel radiation technique called volumetric intensity modulated arc therapy (VMAT) was introduced., ${ }^{3} 4$ The VMAT systems can deliver a highly conformal radiation dose to the target by allowing the simultaneous variation of gantry rotation speed, dose rate and positions of multiple-leaf collimators (MLC). ${ }^{3,4}$

Several authors have conducted the evaluation of dose calculation algorithms for external beam radiation therapy. ${ }^{5-16}$ Rana et $a l .{ }^{16}$ investigated the dose prediction accuracy of Acuros XB algorithm and anisotropic analytical algorithm (AAA) for different field sizes and air gap thicknesses. The results from that study ${ }^{16}$ revealed that dose predictions errors up to $3.8 \%$ for Acuros $\mathrm{XB}$ and up to $10.9 \%$ for AAA could occur during radiation treatment. Furthermore, the study by Rana et al. ${ }^{16}$ demonstrated the limitation of dose calculation algorithms when treating a smaller size of tumor, especially when larger air gaps are created by immobilization devices. The motivation of our study was to further explore the dose 
prediction accuracy of different dose calculation algorithm called collapsed cone convolution superposition (CCCS) algorithm employed in ADAC Pinnacle ${ }^{3} 3 \mathrm{D}$ treatment planning system v. 9.0 (Philips Healthcare, Andover, MA). In this study, we used the similar methodology described by Rana et al. ${ }^{16}$, but we investigated using two air gaps between two solid-water materials. The evaluation of CCCS was done by comparing the percent depth dose (PDD) calculated by CCCS with the measured PDD.

\section{Methods and Materials}

This study utilized a 6 Megavoltage (MV) X-ray beam from ElektaSynergy 1981 linear accelerator (Elekta AB, Stockholm). For all dose computations and measurements, the source to surface distance (SSD) was kept at $100 \mathrm{~cm}$.

\section{Collapsed Cone Convolution Superposition Algorithm}

The CCCS superposition model uses an algorithm in which dose is computed from first principles, thereby accounting for patient heterogeneity and other modifiers. ${ }^{17}$ This is done by modeling the energy fluence of the beam exiting the gantry head, computation of the total energy released per unit mass (TERMA) in the tissue volume, superposing the TERMA with an energy kernel, and accounting for electron contamination which is then added to the photon dose. ${ }^{17-19} \mathrm{~A}$ detailed description on CCCS in provided elsewhere. ${ }^{19}$

\section{Percent Depth Dose Calculation and Measurement}

An inhomogeneous phantom $\left(30 \times 30 \mathrm{~cm}^{2}, 30 \mathrm{~cm}\right.$ deep $)$ composed of rectangular solid-water blocks and two $5 \mathrm{~cm}$ air gaps [Figure 1] was manufactured and scanned using Siemens Somatom Sensation Open CT (Siemens Medical Solutions USA, Inc., Malvern, PA).

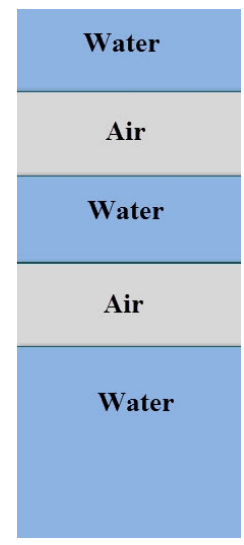

FIG. 1: Schematic diagram of an inhomogeneous phantom. The bottom or fifth layer (water medium) was $10 \mathrm{~cm}$ in thickness, whereas other four layers were each $5 \mathrm{~cm}$ in thickness.
The CT data set of phantom was transferred to the Pinnacle TPS from which a 3D structure set was created. The central axis depth dose calculations were then performed using CCCS for open field sizes $3 \times 3 \mathrm{~cm}^{2}, 5 \times 5 \mathrm{~cm}^{2}, 10 \times 10 \mathrm{~cm}^{2}$, and $15 \times 15 \mathrm{~cm}^{2}$, and for 100 monitor units (MUs). The dose calculation grid size was set to $4 \mathrm{~mm}$.

At selected depths in the water medium of inhomogeneous phantom, measurements were performed using cylidnrical ionization chamber (PTW TN30013, $0.6 \mathrm{~cm}^{3}$ sensitive volume) for identical beam parameters and same number of MUs as in the depth dose calculations. The measurements at each depth were repeated three times. The calculated and measured depth doses were then normalized to the dose obtained at the depth of $1.7 \mathrm{~cm}$. The difference $(\Delta)$ between percent depth dose (PDD) computed by CCCS and the measured PDD was calculated by using Equation 1 .

$$
\Delta\left(P D D_{d}\right)=\frac{C C C S-M E A S}{M E A S} \times 100 \quad \text { Eq. } 1
$$

where, PDD $d=$ percent depth dose at depth, $\mathrm{d}$; CCCS $=$ collapsed cone convolution superposition; MEAS = measurement.

\section{Results}

The measured PDDs and calculated PDDs are presented in Figure 2 for field sizes $3 \times 3 \mathrm{~cm}^{2}, 5 \times 5 \mathrm{~cm}^{2}, 10 \times 10 \mathrm{~cm}^{2}$, and $15 \times 15 \mathrm{~cm}^{2}$.

\section{First Water Medium}

In the first water medium, the CCCS predicted the PDD within $\pm 1.4 \%$ of measured PDD. The highest dose prediction error (up to $-1.4 \%$ ) was obtained for the smallest test field size.

\section{Second Water Medium}

In the second water medium (i.e., after the first air gap), the CCCS under-predicted the PDD at all depths for all four test field sizes. Specifically, dose prediction errors ranged from $-1.6 \%$ to $-3.3 \%$ for $3 \times 3 \mathrm{~cm}^{2}$, from $-2.4 \%$ to $-4.2 \%$ for $5 \times 5$ $\mathrm{cm}^{2}$, from $-2.4 \%$ to $-6.7 \%$ for $10 \times 10 \mathrm{~cm}^{2}$, and from $-1.6 \%$ to $-6.3 \%$ for $15 \times 15 \mathrm{~cm}^{2}$.

\section{Third Water Medium}

In the third water medium, the CCCS continued to under-predict the PDDs at all depths for all test field sizes. Specifically, dose prediction errors ranged from $-3.2 \%$ to $-3.9 \%$ for $3 \times 3 \mathrm{~cm}^{2}$, from $-2.4 \%$ to $-5.6 \%$ for $5 \times 5 \mathrm{~cm}^{2}$, from $-2.3 \%$ to $-6.0 \%$ for $10 \times 10 \mathrm{~cm}^{2}$, and from $-1.5 \%$ to $-5.6 \%$ for $15 \times$ $15 \mathrm{~cm}^{2}$. 

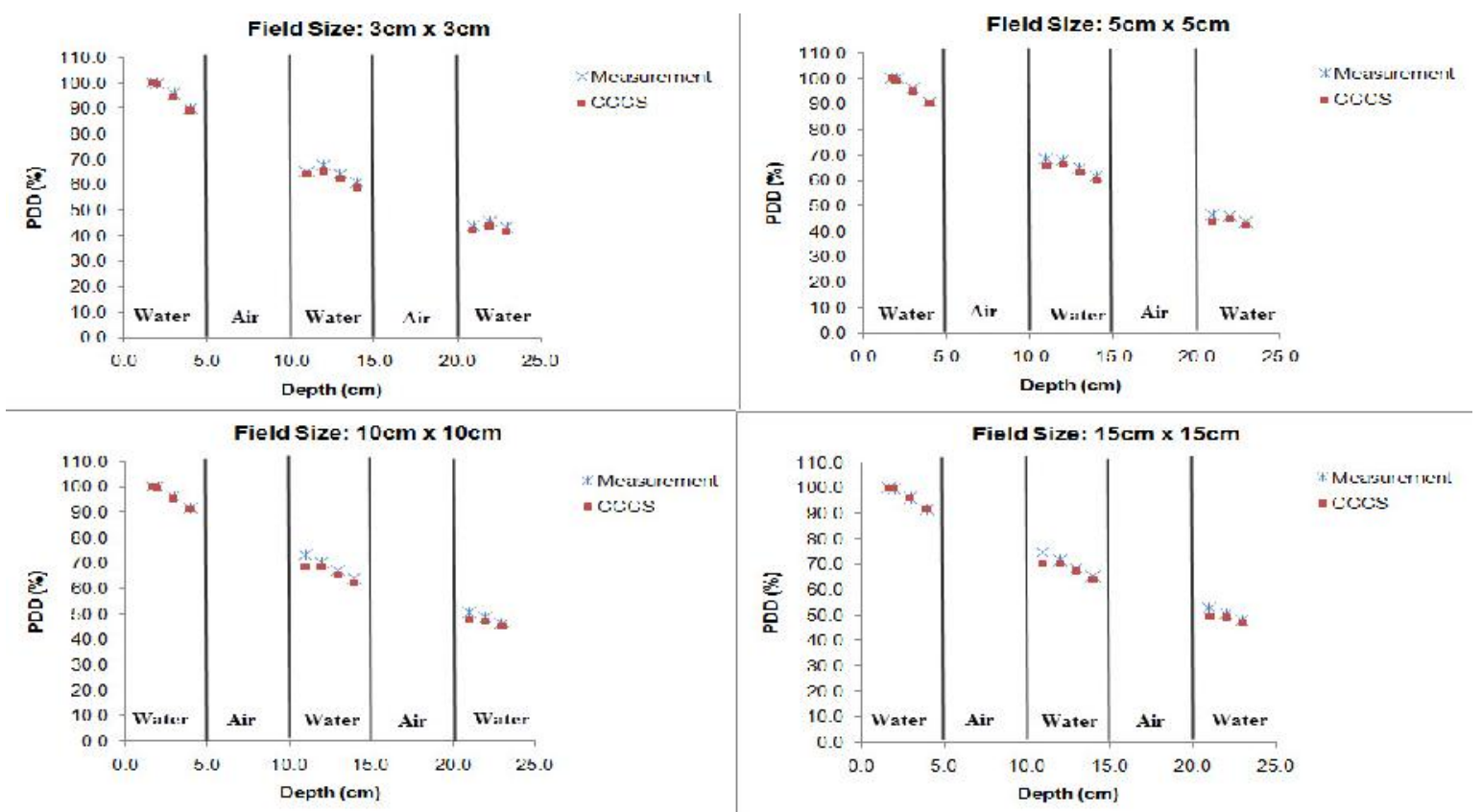

FIG. 2: Calculated and measured PDD for field sizes $3 \times 3,5 \times 5,10 \times 10$, and $15 \times 15 \mathrm{~cm}^{2}$ in inhomogeneous phantom. The first (top), second, third, fourth, and fifth (bottom) layers represent solid-water $(5 \mathrm{~cm})$, air gap $(5 \mathrm{~cm})$, solid-water $(5 \mathrm{~cm})$, air gap $(5 \mathrm{~cm})$ and solid-water $(10 \mathrm{~cm})$ media, respectively, in the phantom. The measurements were done at SSD of $100 \mathrm{~cm}$ using $6 \mathrm{MV}$ photon beam for $100 \mathrm{MUs}$, and all dose calculations and measurements were normalized to the reading obtained at depth of maximum dose $\left(\mathrm{d}_{\max }=1.7 \mathrm{~cm}\right) . \quad A b b r e v i a t i o n s: ~ P D D=$ Percent Depth Dose, CCCS = Collapsed Cone Convolution Superposition

\section{Discussion}

In this study, dose calculation accuracy of CCCS has been evaluated by comparing the calculated and measured PDD at multiple depths in an inhomogeneous slab phantom containing two air gaps. Although the CCCS had good agreement with the measurement in the first water medium, the results showed the limitation of CCCS in predicting doses in second water medium (i.e., after the first air gap) as well as in the third water medium (i.e., after the second air gap). As the photon beam traverses the air gap, loss of lateral scatter increases within the air gap, and this causes decreased scatter dose contribution to the points along the central beam axis. Furthermore, media of different density can cause the electronic disequilibrium at and near their heterogeneity interface. ${ }^{5}$ Thus, dose discrepancies seen in the water media after the air gaps may be due to improper beam modeling within CCCS.

In this study, dose prediction accuracy of CCCS was investigated using low-density medium only. However, in real clinical situations, photon beams may also pass through the high-density tissues/materials before reaching the target. Future work involves the dosimetric evaluation of CCCS in inhomogeneous phantom that is composed of high- and low density materials such as bone and lung tissues. The limitation of CCCS must be further investigated in different clinical scenarios in order to avoid the dose overestimation or underestimation when CCCS is used for dose computations in external beam radiation therapy planning.

\section{Conclusion}

The results of this study showed that the CCCS under-predicted the depth doses in the water medium after the photon beam traversed the air gaps. Special attention must be given during the patient set-up since large air gap between the patient body and immobilization devices may lead to unacceptable dose prediction errors.

\section{Conflict of interest}

The authors declare that they have no conflicts of interest. The authors alone are responsible for the content and writing of the paper.

\section{References}

1. Webb S. Intensity-modulated radiation therapy. Bristol: Institute of Physics Publishing 2000. 
2. Guerrero Urbano MT, Nutting CM. Clinical use of intensity-modulated radiotherapy: part I. $\mathrm{BrJ}$ Radiol 2004; 77:88-96.

3. Otto K. Volumetric modulated arc therapy: IMRT in a single gantry arc. Med Phys 2008; 35: 310-317.

4. Teoh M, Clark CH, Wood K, Whitaker S, Nisbet A. Volumetric modulated arc therapy: A review of current literature and clinical use in practice. $B r J$ Radiol 2011; 84: 967-96.

5. Das IJ, Ding GX, Ahnesjö A. Small fields: Non-equilibrium radiation dosimetry. Med Phys 2008; 35: 206-15.

6. Robinson D. Inhomogeneity correction and the analytic anisotropic algorithm. J Appl Clin Med Phys 2008; 9:2786.

7. Ding $\mathrm{W}$, Johnston $\mathrm{P}, \mathrm{Wong} \mathrm{T}$, et al. Investigation of photon beam models in heterogeneous media of modern radiotherapy. Australas Phys Eng Sci Med 2004; 27: 39-48.

8. Carrasco P, Jornet N, Duch M, et al. Comparison of dose calculation algorithms in phantoms with lung equivalent heterogeneities under conditions of lateral electronic disequilibrium. Med Phys 2004; 31: 2899-2911.

9. Krieger T, Sauer OA. Monte Carlo- versus pencil-beam-/collapsed-cone-dose calculation in a heterogeneous multi-layer phantom. Phys Med Biol 2005; 50:859-68.

10. Van Esch A, Tillikainen L, Pyykkonen, et al. Testing of the analytical anisotropic algorithm for photon dose calculation. Med Phys 2006; 33: 4130-48.

11. Dobler B, Walter C, Knopf A, Fabri D, Loeschel R, Polednik M, Schneider F, Wenz F, Lohr F. Optimization of extracranial stereotactic radiation therapy of small lung lesions using accurate dose calculation algorithms. Radiat Oncol 2006; 1:45.

12. Vanderstraeten B, Reynaert N, Paelinck L, Madani I, De Wagter C, De Gersem W, De Neve W, Thierens H. Accuracy of patient dose calculation for lung IMRT: A comparison of Monte Carlo, convolution/superposition, and pencil beam computations. Med Phys 2006; 33:3149-58.
13. Gray A, Oliver LD, Johnston PN. The accuracy of the pencil beam convolution and anisotropic analytical algorithms in predicting the dose effects due to attenuation from immobilization devices and large air gaps. Med Phys 2009; 36:3181-3191.

14. Rana S, Rogers K, Lee T, Reed D, Biggs C. Verification and Dosimetric Impact of Acuros XB Algorithm for Stereotactic Body Radiation Therapy (SBRT) and RapidArc Planning for Non-Small-Cell Lung Cancer (NSCLC) Patients. Int JMed Phys Clin Eng Rad Onc 2013; 2: 6-14.

15. Han T, Mourtada F, Kisling K, Mikell J, Followill D, Howell R. Experimental validation of deterministic Acuros XB algorithm for IMRT and VMAT dose calculations with the Radiological Physics Center's head and neck phantom. Med Phys 2012; 39: 2193-2202.

16. Rana S, Rogers K. Dosimetric evaluation of Acuros $\mathrm{XB}$ dose calculation algorithm with measurements in predicting doses beyond different air gap thickness for smaller and larger field sizes. JMed Phys 2013; 38:9-14.

17. Ahnesjö A. Collapsed cone convolution of radiant energy for photon dose calculation in heterogeneous media. Med Phys 1989; 16:577-92.

18. Mackie TR, Scrimger JW, Battista JJ. A convolution method of calculating dose for 15-MV X rays. Med Phys 1985; 12:188-96.

19. McNutt T. Dose calculations: collapsed cone convolution superposition and delta pixel beam. 2002: Pinnacle White Paper No. 453598302474. 